\title{
Synthesis and Properties of Benzo[a]phenoxazinium Chalcogen Analogues as Novel Broad-Spectrum Antimicrobial Photosensitizers
}

\author{
James W. Foley ${ }^{\star}, \dagger$, Xiangzhi Song $^{\dagger}$, Tatiana N. Demidova $¥, \S$, Fatima Jilal ${ }^{\ddagger}, \|$, and Michael \\ R. Hamblin ${ }^{*} \ddagger, \perp, \#$ \\ ${ }^{\dagger}$ Rowland Institute at Harvard, Harvard University, Cambridge, Massachusetts 02142 \\ $\ddagger$ Wellman Center for Photomedicine, Massachusetts General Hospital, Boston, Massachusetts \\ 02114 \\ $\S$ Graduate Program in Cell Molecular and Developmental Biology, Sackler School of Graduate \\ Biomedical Sciences, Tufts University School of Medicine, Boston, Massachusetts 02111 \\ "Aga Khan Medical School, Karachi, Pakistan \\ ${ }^{\perp}$ Department of Dermatology, Harvard Medical School, Boston, Massachusetts 02115 \\ \#Harvard-MIT Division of Health Sciences and Technology, 77 Massachusetts Avenue E25-519, \\ Cambridge, Massachusetts 02139
}

\begin{abstract}
The goal of this investigation was to develop improved photosensitizers for use as antimicrobial drugs in photodynamic therapy of localized infections. Replacement of the oxygen atom in 5(ethylamino)-9-diethylaminobenzo[ $a]$ phenoxazinium chloride (1) with sulfur and selenium afforded thiazinium and selenazinium analogues $\mathbf{2}$ and $\mathbf{3}$, respectively. All three dyes are water soluble, lipophilic, and red light absorbers. The relative photodynamic activities of the chalcogen series were evaluated against a panel of prototypical pathogenic microorganisms: the Grampositive Enterococcus faecalis, the Gram-negative Escherichia coli, and the fungus Candida albicans. Selenium dye $\mathbf{3}$ was highly effective as a broad-spectrum antimicrobial photosensitizer with fluences of 4-32 J/cm² killing 2-5 more logs of all cell types than sulfur dye 2 , which was slightly more effective than oxygen analogue $\mathbf{1}$. These data, taken with the findings of uptake and retention studies, suggest that the superior activity of selenium derivative $\mathbf{3}$ can be attributed to its much higher triplet quantum yield.
\end{abstract}

\section{Introduction}

Photodynamic therapy (PDT ${ }^{\mathrm{a}}$ ) is a medical treatment that uses the energy of actinic light to selectively inactivate diseased tissues and infectious pathogens. ${ }^{1}$ Therapy involves the systemic or topical administration of a target localizing, nontoxic dye or photosensitizer (PS) that becomes, or in reaction with molecular oxygen generates, a cytotoxin when illuminated

\footnotetext{
(C) 2006 American Chemical Society

"Corresponding author. Phone: 617-497-4677. Fax: 617-497-4627. foley@ rowland.harvard.edu (J.W.F.). Phone: 617-716-6182. Fax: 617-726-8566. hamblin@helix.mgh.harvard.edu (M.R.H.). .

Supporting Information Available: Elemental analysis of key compound $\mathbf{3}$ and Figures showing photodynamic inactivation of $E$. faecalis using photosensitizers 2, 3, and E. coli using PS $\mathbf{3}$ with and without a wash. This material is available free of charge via the Internet at http://pubs.acs.org.
} 
with light of the appropriate wavelength. ${ }^{2,3}$ Because the power and safety of the method derive from its dual selectivity, that is, inactivation occurs only where the PS and light are simultaneously present, effective treatment relies on the availability of PSs having a high degree of specificity and affinity for selected biological targets. ${ }^{4}$ The discovery of PDT dyes having these characteristics continues to be the focus of considerable interest and research activity. ${ }^{5}$

The concept of PDT is not new, having been practiced since at least the 15th century in the Middle East, where ingested naturally occurring psoralen PS and sunlight were used to treat vitiligo. ${ }^{6}$ Although PDT was rediscovered over 100 years ago as a means for killing microorganisms, it remained a laboratory curiosity until the 1960s when the method was shown to be a safe and efficacious treatment for solid tumors in humans. ${ }^{7,8}$ However, under the scrutiny of experimental and clinical evaluation, the PSs used in these studies, hematoporphyrin derivative (HPD) and Photofrin (a purified fraction of HPD), were found to suffer from several drawbacks: (a) both have low coefficients of absorption in the therapeutic window, the spectral region where light penetrates tissue maximally (600-900 $\mathrm{nm}$ ); (b) both consist of a complex mixture of porphyrin ether and ester monomers and oligomers; and most significantly, (c) they both cause prolonged cutaneous photosensitization. ${ }^{9}$ Mechanistically, these drugs cause tumor destruction primarily by shutting down the microvasculature supporting the tumor and to a lesser extent by direct photosensitization of the tumor cells. ${ }^{10}$

The demonstrated potential of PDT as a powerful new modality for treating localized neoplasia, coupled with the recognized limitations of HPD and Photofrin, provided strong incentives for the development of more efficacious second generation PDT agents. Owing to the successes reported regarding the use of HPD, it is perhaps not surprising that most attempts to design these improved drugs have centered on members of the extended porphyrin family possessing a tetrapyrrole backbone (i.e., chlorins, purpurins, phthalocyanines, etc.). ${ }^{5}$ Less consideration has been paid to an attractive, contrasting approach that is based on the hypothesis that drugs derived from structurally dissimilar families with inherently different physicochemical and pharmacological properties would exhibit favorable efficacy, toxicity, and mechanistic profiles and, as a result, prove to be complementary to one another. To this end, we have investigated the PDT properties of a series of dyes belonging to the benzophenoxazinium family of cationic chromophores. ${ }^{11,12}$ We were drawn to this class of dyes because Lewis et al. had reported that the textile dye Nile Blue A (NBA) and a select set of derivatives, when administered orally, strongly stained solid murine tumors while imparting little or no coloration to the surrounding

$\begin{array}{ll}\text { aAbbreviations: } & \\ \text { EtNBA } & \text { 5-(ethylamino)-9-diethylaminobenzo }[a] \text { phenoxazinium chloride } \\ \text { EtNBS } & \text { 5-(ethylamino)-9-diethylaminobenzo }[a] \text { phenothiazinium chloride } \\ \text { EtNBSe } & \text { 5-(ethylamino)-9-diethylaminobenzo }[a] \text { phenoselenazinium chloride } \\ \text { HPD } & \text { hematoporphyrin derivative } \\ \text { NBA } & \text { nile blue A } \\ \text { PDI } & \text { photodynamic inactivation } \\ \text { PDT } & \text { photodynamic therapy } \\ \text { PS } & \text { photosensitizer } \\ \text { SDS } & \text { sodium dodecyl sulfate }\end{array}$


healthy tissues; significantly, the dyes appeared to be relatively innocuous, were efficient absorbers of 600-700 $\mathrm{nm}$ light, and were rapidly eliminated ( $24 \mathrm{~h}$ post administration). ${ }^{13,14}$

Although an investigation of the PDT potential of the Lewis dyes found them to be inefficient PSs both in vitro and in vivo, they nonetheless proved to be a valuable starting point for the rational design of drugs with improved photoactivities. One of the most effective anticancer PDT agents discovered early in this program involved the replacement of the $\mathrm{O}$ atom of an ethylated-NBA analogue (1, EtNBA) with a $\mathrm{S}$ atom to give the novel PS 5-(ethylamino)-9-diethylaminobenzo[ $a$ ]phenothiazinium chloride (2, EtNBS). ${ }^{12}$ This drug, when activated with $650 \mathrm{~nm}$ light, showed good efficacy against several murine sarcomas (EMT-6, RIF), a human carcinoma (FaDu), and a rat glioma (9L); ${ }^{15}$ in the case of one study using the EMT-6 model, $100 \%$ of the animals showed a complete response. ${ }^{16}$ Mechanistic studies revealed that EtNBS (2) rapidly accumulated in cancer cells and, unlike HPD, caused inactivation primarily by directly killing the tumor cells. ${ }^{16}$ An evaluation of this PDT drug culminated in a preclinical study that found EtNBS (2)-PDT to be highly effective against selected spontaneous tumors in pet cats and dogs. ${ }^{17}$ In general, the drug was well tolerated and caused no residual photosensitization 5 days post-treatment for both species.

We attributed the higher level of phototoxicity shown by EtNBS (2) compared to that of its $\mathrm{O}$ analogue EtNBA (1) to the heavy atom effect, wherein the increased spin-orbital coupling constants associated with atoms having high atomic numbers increases excited triplet state formation, the species thought to mediate cytotoxicity. ${ }^{6}$ Consequently, as an extension of this strategy, we were motivated to synthesize EtNBSe (3), a chalogen analogue having a still heavier Se atom in place of the centrally located S atom of EtNBS (2). In an in vitro study using the EMT-6 mammary sarcoma cell line, a comparative phototoxicity evaluation of EtNBSe (3) with EtNBS (2), EtNBA (1), and Photofrin (all at $0.5 \mathrm{mM}$ extracellular concentration) found that the light dose required to kill $50 \%$ of the cells was $<0.5 \mathrm{~J} / \mathrm{cm}^{2}$ for EtNBSe (3) and $2.0 \mathrm{~J} / \mathrm{cm}^{2}$ for EtNBS (2), whereas the dose to achieve the same response for EtNBA (1) and Photofrin could not be determined because of their low phototoxicities. ${ }^{18}$ The relative photoactivities of the three members of the chalogen series correlated with their abilities to generate single oxygen and with limited intracellular uptake for Photofrin. Thus, although never studied as rigorously as EtNBS (2), the data gathered for EtNBSe (3) suggests that it has the potential to be a superior PDT drug.

Because of the well-known increase in multi-antibiotic resistance among pathogenic microbes of all classes, PDT has attracted growing attention as a possible treatment for localized infections. ${ }^{19}$ Preclinical studies have found that many commonly used porphyrin PSs are effective at inactivating Gram-positive ${ }^{20}$ bacteria but are considerably less efficacious against Gram-negative organisms; ${ }^{21}$ fungal cells such as Candida are generally midway in susceptibility between both types of bacteria. ${ }^{22}$ Cationic PSs, however, have been found to be effective against both Gram-positive and Gram-negative bacteria, ${ }^{23}$ which is consistent with the observation that most dyes used in pathology to identify bacteria and bacterial spores bear a delocalized cationic charge as exemplified by the Gram stain/counterstain pair, Gentian Violet and Fuchsin, ${ }^{24}$ and the Wirtz-Conklin pair, Malachite Green and Safranine-O. ${ }^{25}$ For example, Methylene Blue, Toluidine Blue and Dimethylmethylene Blue, phenothiazinium dyes closely related to EtNBS (2), are safe and effective antimicrobial $\mathrm{PSs}^{26}$ that are currently the only PDT agents being used for clinical antimicrobial applications in the oral cavity for such indications as periodontitis ${ }^{27}$ and tooth cavity sterilization. ${ }^{28}$ Nevertheless, the use of these dyes suffers constraints that might impair their activities. First, they must be used in relatively high concentrations to achieve multiple logs of cell killing with reasonable light fluences. Second, they are much more effective when the dye remains present in the incubation mixture during illumination, compared to that when the microbes are washed free from unbound dye before light delivery. ${ }^{22}$ Thus, further efforts 
need to be directed to the development of more tenacious, active cationic antibacterial PDT drugs.

The main purpose of this article is to present the results of a study designed to evaluate the broad-spectrum antimicrobial sensitizing efficacies of a series of three chalcogen $(\mathrm{O}, \mathrm{S}, \mathrm{Se})$ substituted benzo[ $a]$ phenoxazinium analogues against a panel of prototypical human pathogenic microbes consisting of the Gram-positive bacterium Enterococcus faecalis, the Gram-negative bacterium Escherichia coli, and the yeast Candida albicans. We relate these findings to pertinent physical and photophysical properties of all three chalcogen dyes.

Additionally, because EtNBSe (3) is the first and only benzo[ $a]$ phenoselenazinium dye that has been described in the literature, we herein present the synthetic approach used to prepare this novel PS.

\section{Experimental Section}

\section{Chemical Synthesis}

All solvents were reagent grade and used as received. 5-Ethylamino-9diethylaminobenzo[a]phenoxazinium (1, EtNBA) and 5-(ethylamino)-9diethylaminobenzo[ $[a]$ phenothiazinium (2, EtNBS) were available from earlier studies and had been prepared using previously described procedures. ${ }^{29-31}$ All intermediates and dyes were purified using medium pressure (100 psi) chromatography using Woelm 32-63 silica gel. Thin-layer chromatography was performed using commercially prepared silica gel covered glass plates (Whatman K5F 150A). UV-visible absorption spectra were recorded using an HP 8453 spectrophotometer. Corrected fluorescence spectra were recorded using a SPEX Fluorolog2 (solvent measurements) or a FluoroMax3 (microorganism measurements), SPEX Industries, Edison, NJ; fluorescence quantum yields were measured relative to the Cresyl Violet standard at $25^{\circ} \mathrm{C} .{ }^{32}$ Quantum yields for ${ }^{1} \mathrm{O}_{2}$ formation were determined by measuring the ${ }^{1} \mathrm{O}_{2}$-mediated bleaching of 1,3-diphenylisobenzofuran ${ }^{12}$ relative to Methylene Blue $(0.50)^{33}$ at $25^{\circ} \mathrm{C} .{ }^{1} \mathrm{H}$ NMR spectra were obtained using a Varian $400 \mathrm{MHz}$ spectrometer with TMS as the internal standard; chemical shifts are reported in $\delta$, coupling constants as $J$ (cps) using standard peak splitting terminology. HRMS (electrospray ionization (ESI)) were obtained from the Mass Spectroscopy Facility, Chemical and Chemical Biology Department, Harvard University.

\section{Bis-(3- $N, N$-diethylaminophenyl) Diselenide (5)}

To magnesium turnings $(1.94 \mathrm{~g}, 80 \mathrm{mmol})$ stirred in fresh, dry ethyl ether $(50 \mathrm{~mL})$ under an argon atmosphere was added dropwise over a $3 \mathrm{~h}$ period an ethyl ether $(50 \mathrm{~mL})$ solution of 3-iodo- $N, N$-diethylaniline ${ }^{34}(4)(10.0 \mathrm{~g}, 36.4 \mathrm{mmol})$ and 1,2-dibromoethane $(6.84 \mathrm{~g}, 36.4$ $\mathrm{mmol}$ ). After an additional hour of stirring, most of the magnesium had reacted. To the resulting solution, Grignard reagent was slowly added, via a flexible solids addition tube, selenium powder ( $3.35 \mathrm{~g}, 42.4 \mathrm{mmol}, 325 \mathrm{mesh}$ ); the addition rate (15 min) was modulated so as to keep a controlled, gentle solvent reflux, which results from the exotherm of the reaction. The resulting mixture was stirred overnight at room temperature. The reaction was carefully quenched with the dropwise addition of $30 \mathrm{~mL}$ of water (initial vigorous reaction) to give a dark orange ether layer and a brown water layer. Air was bubbled through this stirred mixture overnight; the exhaust, which contained selenium vapor (TOXIC), was trapped by bubbling through a solution of aqueous sodium hypochlorite (bleach). The residue was taken up in ethyl ether $(250 \mathrm{~mL})$ and washed with water and brine and dried over sodium sulfate. Removal of the solvent in vacuo afforded $7.35 \mathrm{~g}$ of a yellow oil that was purified using silica gel chromatography using $1 \%$ ethyl ether in hexane as eluent to afford $4.61 \mathrm{~g} \mathrm{(56 \% )}$ of diselenide 5 as a light yellow oil. TLC analysis indicated that the product contained a trace of a single impurity that was below the detection limits of NMR 
spectroscopy; 5 was used without further purification. ${ }^{1} \mathrm{H} \mathrm{NMR}\left(\delta / \mathrm{ppm}, \mathrm{CDCl}_{3}\right): 1.10(\mathrm{t}, J=$ $7.2,12 \mathrm{H}), 3.28(\mathrm{q}, J=7.2,8 \mathrm{H}), 6.51(\mathrm{dd}, J=7.6,2.0,2 \mathrm{H}), 6.90(\mathrm{~m}, 4 \mathrm{H}), 7.06(\mathrm{dd}, J=8.4$, 7.6, 2H). HRMS (ESI) $m / z \mathrm{C}_{20} \mathrm{H}_{28} \mathrm{~N}_{2} \mathrm{Se}_{2}$ (MH+) calcd, 457.0661; found, 457.0655.

\section{Bis-(3-N,N-diethylamino-6-nitrosophenyl) Diselenide (6)}

To a stirred, cold $\left(5^{\circ} \mathrm{C}\right)$ solution of diselenide $5(2.36 \mathrm{~g}, 5.2 \mathrm{mmol})$ in $1 \mathrm{~N}$ hydrochloric acid $(200 \mathrm{~mL})$ was added an aqueous solution of sodium nitrite $(0.72 \mathrm{~g}, 11.4 \mathrm{mmol})$, whereupon the clear yellow solution rapidly formed an orange precipitate. After an additional 10 min of stirring, the mixture was extracted twice with methylene chloride $(200 \mathrm{~mL})$. The organic layer was washed twice with brine and dried over sodium sulfate. The solvent was removed in vacuo to give $2.22 \mathrm{~g}$ of an orange/brown solid. Crystallization from 2-propanol gave 1.84 $\mathrm{g}(69 \%)$ of brown crystals. TLC analysis (5\% methanol in methylene chloride) showed that the desired product contained a trace amount of a blue contaminant that could not be removed by an additional crystallization. Thus, 6 was used without further purification. ${ }^{1} \mathrm{H}$ $\operatorname{NMR}\left(\delta / \mathrm{ppm}, \mathrm{CDCl}_{3}\right): 1.43$ (broad m, 12H), 3.70 (broad m, 8H), $7.13(\mathrm{dd}, J=9.6,2.4,2 \mathrm{H}$ ), $7.84(\mathrm{~d}, J=2.44 \mathrm{H}), 8.38(\mathrm{~d}, J=9.6,2 \mathrm{H})$. HRMS (ESI) $m / z \mathrm{C}_{20} \mathrm{H}_{26} \mathrm{~N}_{4} \mathrm{O}_{2} \mathrm{Se}_{2}(\mathrm{MH}+$ ) calcd, 515.0464; found, 515.0458.

\section{5-Ethylamino-9-diethylaminobenzo[a]phenoselenazinium Chloride (3, EtNBSe)}

A stirred solution of bis-nitroso diselenide $(6)(1.70 \mathrm{~g}, 3.3 \mathrm{mmol})$ and $1-\mathrm{N}$ ethylnaphthylamine (7) $(1.49 \mathrm{~g}, 9.6 \mathrm{mmol})$ in trifluoroethanol was heated to reflux temperature. Initially, the reaction solution showed the formation of an absorption band at $810 \mathrm{~nm}$ that was rapidly replaced by a deep blue band at $660 \mathrm{~nm}$. Reaction was complete in less than $1 \mathrm{~h}$, whereupon the solvent was removed in vacuo to give a blue waxy solid. The solid was stirred twice with ethyl ether to remove excess 7 and dissolved in a mixture of aqueous $1 \mathrm{~N}$ sodium hydroxide and methylene chloride. The color of the solution turned bright magenta, indicating that deprotonation of $\mathbf{3}$ had occurred. The mixture was placed in a separatory funnel, and the organic layer was washed twice with brine. Dye $\mathbf{3}$ was regenerated as a deep blue chloride salt by adding $0.5 \mathrm{~mL}$ of concentrated hydrochloric acid to the magenta methylene chloride solution. After removing the solvent and excess hydrochloric acid in vacuo, the desired product was purified by column chromatography using a solvent gradient of methanol $(2-7 \% \mathrm{v} / \mathrm{v})$ in methylene chloride. Fractions that consisted of a single spot by TLC analysis were combined to give $\mathbf{3}(1.49 \mathrm{~g}, 55 \%)$ as a noncrystalline solid. Because $\mathbf{3}$ binds tenaciously to a trace amount of solvent (NMR analysis), which we were unable to remove using high vacuum and heat, the dye was twice dissolved in anhydrous ethanol and evaporated to dryness in order to exchange the residual eluting solvent with this innocuous alcohol. A sample for combustion analysis was prepared by adding a small amount of water to a methanol/methylene chloride $(1: 15, \mathrm{v} / \mathrm{v})$ solution of the dye and removing the solvent under vacuum with gentle heating to constant weight. Anal. $\left(\mathrm{C}_{22} \mathrm{H}_{24} \mathrm{~N}_{3} \mathrm{SeCl}-0.5 \mathrm{H}_{2} \mathrm{O}\right) \mathrm{C}, \mathrm{H}, \mathrm{N} .{ }^{1} \mathrm{H}$ NMR $\left(\delta / \mathrm{ppm}, \mathrm{MeOH}-\mathrm{d}_{4}\right): 1.32$ (t, $J=6.8,6 \mathrm{H}$, $\left.\mathrm{N}\left(\mathrm{CH}_{2} \mathrm{CH}_{3}\right)_{2}\right), 1.44\left(\mathrm{t}, J=7.2,3 \mathrm{H}, \mathrm{NH}\left(\mathrm{CH}_{2} \mathrm{CH}_{3}\right)\right.$ ), 3.65 (two overlapping q, $J=7.2$ and 6.8 , $6 \mathrm{H}$, both types of $\left.\mathrm{N}-\mathrm{CH}_{2}\right), 7.13(\mathrm{dd}, J=9.6,2.4,1 \mathrm{H}), 7.40(\mathrm{~d}, J=2.8,1 \mathrm{H}), 7.55(\mathrm{~s}, 1 \mathrm{H})$, $7.74(\mathrm{~m}, 1 \mathrm{H}), 7.82(\mathrm{~m}, 1 \mathrm{H}), 7.96(\mathrm{~d}, J=9.6,1 \mathrm{H}), 8.21(\mathrm{~d}, J=8.41 \mathrm{H}), 8.99(\mathrm{~d}, J=8.4,1 \mathrm{H})$. HRMS (ESI) $m / z \mathrm{C}_{22} \mathrm{H}_{24} \mathrm{~N}_{3} \mathrm{Se}(\mathrm{M}+$ ) calcd, 410.1135; found, 410.1138 .

\section{Photostability of Photosensitizers}

To ensure that the PSs of the present study, especially selenium derivative $\mathbf{3}$, are resistant to photodegradation by singlet oxygen, a water-cooled $1 \mathrm{~cm}^{2}$ square cuvette containing $3 \mathrm{~mL}$ of a methanol/acetic acid; $(100: 1, \mathrm{v} / \mathrm{v})$ solution of each dye $(\mathrm{OD}=1.0)$ was placed in a cell holder. One face of the cuvette was subjected to the unfiltered beam emanating from a slide projector (Polaroid 610, fluence $72 \mathrm{Jcm}^{-2}$ at an irradiance of $20 \mathrm{mWcm}^{-2}$ for the $600-700$ $\mathrm{nm}$ band). The optical density and the wavelength of maximum absorption of each solution 
were compared before and after illumination; in all cases, the OD of the solutions decreased less than $4 \%$, whereas the wavelengths of maximum absorption, a sensitive probe for detecting changes at the 7 chalcogen-substituted ring position, ${ }^{35}$ remained unchanged, indicating that at least in the relatively benign environment afforded by ethanol all of the dyes are relatively stable.

\section{Microbial Strains}

The microorganisms studied were Escherichia coli (ATCC 25922), Enterococcus faecalis (ATCC 29212), and Candida albicans (ATCC 18804). Cells were grown at $37^{\circ} \mathrm{C}$ in aerobic conditions in a shaker set at $150 \mathrm{rpm}$. Brain-heart infusion broth (Difco, BD Diagnostic Systems, Sparks, MD) was used for E. coli and E. faecalis, and YM medium (Difco) was used for $C$. albicans. Exponential cultures obtained by reculturing stationary overnight precultures were used for all experiments. E. coli and E. faecalis were grown in a fresh medium for approximately $1 \mathrm{~h}$ to a density of $10^{8}$ cells $/ \mathrm{mL}$; the OD values at $650 \mathrm{~nm}$ were 0.6. C. albicans was grown for approximately $4 \mathrm{~h}$ to an approximate density of $10^{7}$ cells $/ \mathrm{mL}$ corresponding to an OD of 6 at $650 \mathrm{~nm}$ (10 fold dilution measured). Exact cell numbers were confirmed by counting colony forming units obtained after serial dilution on square BHI (or YM for Candida) agar plates.

\section{Photosensitizer Solutions and Light Sources}

Stock solutions $(2 \mathrm{mM})$ of the three chalcogen dyes were prepared in water and stored at 4 ${ }^{\circ} \mathrm{C}$ in the dark for no longer than 1 month before use. A noncoherent light source with interchangeable fiber bundles (LumaCare, London, U.K.) was employed. Band-pass filters $(30 \mathrm{~nm})$ allowed total powers of roughly $1 \mathrm{~W}$ to be obtained from $630 \pm 15 \mathrm{~nm}$ for EtNBA (1), $652 \pm 15 \mathrm{~nm}$ for EtNBS (2), and $660 \pm 15 \mathrm{~nm}$ for EtNBSe (3).

\section{Dye Uptake by Microbial Cells}

Suspensions of microorganisms were incubated for 10 min with PSs in the dark at room temperature. Unbound PSs were washed out by centrifugation of the mixture of dye and microorganisms for $6 \mathrm{~min}$ at $1550 \mathrm{~g}$ followed by resuspension of washed pellets in $10 \mathrm{~mL}$ of PBS without $\mathrm{Ca}^{2+} / \mathrm{Mg}^{2+}$. Aliquots $(200 \mu \mathrm{L})$ of these suspensions were used for photodynamic inactivation (PDI) experiments, the remaining suspensions were centrifuged again, and the pellets were dissolved in $3 \mathrm{~mL}$ of $10 \%$ SDS for at least $24 \mathrm{~h}$. These suspensions were used for PS uptake measurement. The fluorescence of dissolved pellets was measured on a spectrofluorimeter (FluoroMax3, SPEX Industries, Edison, NJ). For EtNBA (1), the excitation wavelength was $625 \mathrm{~nm}$, and the emission spectra of the solution were recorded from 630 to $750 \mathrm{~nm}$. For EtNBS (2), the excitation wavelength was $645 \mathrm{~nm}$, and the range for emission was 650 to $750 \mathrm{~nm}$. For EtNBSe (3), the excitation wavelength was $655 \mathrm{~nm}$, and the emission was recorded in the range from 660 to $750 \mathrm{~nm}$. The fluorescence was calculated from the height of the peaks recorded. Calibration curves were made from pure PS dissolved in 10\% SDS and used for determination of sensitizer concentration in the suspension. Uptake values were obtained by dividing the number of nmol of sensitizer in the dissolved pellet by the number of colony forming units obtained by serial dilutions and the number of PS molecules/cell calculated by using Avogadro's number.

\section{Photodynamic Inactivation Studies}

Illumination was performed either before or after excess dye was washed out. Aliquots of $200 \mu \mathrm{L}$ of cell suspension were placed in 96-well plates and illuminated with appropriate light at room temperature. Fluences ranged from 0 to $80 \mathrm{Jcm}^{-2}$ at an irradiance of $60-100$ $\mathrm{mWcm}^{-2}$. Exact power was measured with a laser power meter (model FM/GS, Coherent, 
Santa Clara, CA). During illumination after defined fluences had been delivered, aliquots of $20 \mu \mathrm{L}$ were taken to determine the colony-forming units. The contents of the wells were mixed before sampling. The aliquots were serially diluted 10 -fold in PBS without $\mathrm{Ca}^{2+}$ / $\mathrm{Mg}^{2+}$ to give dilutions of $10^{-1}-10^{-6}$ times the original concentrations and were streaked horizontally on square BHI agar plates as described by Jett et al. ${ }^{36}$ The plates were incubated at $37{ }^{\circ} \mathrm{C}$ overnight. Colonies were counted and survival fraction determined compared to that of the untreated control. Dye in the absence of light and light alone was also used as controls. PSs were generally nontoxic for microorganisms in the dark, and light alone did not cause cell destruction. All experiments were performed in triplicate.

\section{Statistics}

Values are reported as means $\pm \mathrm{SD}$. Differences between means were evaluated by one-way ANOVA in Microsoft Excel (Microsoft Corp., Redmond, WA). Differences between the slopes of killing curves were evaluated using the linear regression analysis function contained in the GraphPad Prism software (GraphPad Software Inc, San Diego, CA). P values of less than 0.05 were considered significant.

\section{Chemistry}

Benzo $[a]$ phenoxazinium and benzo[ $[a]$ phenothiazinum dyes EtNBA (1) and EtNBS (2), available from antecedent studies, had been synthesized in-house using standard procedures. ${ }^{29-31}$ Surprisingly, prior to our work, the literature contained no example of a benzo[ $[a]$ phenoselenazinum dye and only a single reference to an unsymmetrical phenoselenazinium dye, which had been synthesized by Groves et al. via the acid-catalyzed condensation of a $p$-nitroso- $N, N$-dialkylaniline with bis-(3-aminophenyl) diselenide. ${ }^{37}$ Although our initial efforts for preparing EtNBSe (3) using a sequence of reactions identical to those used in this latter work were unsuccessful, a modification of the Groves approach, outlined in Scheme 1, did afford the PS in high yield. The Grignard reagent derived from 3iodo- $N, N$-diethylaniline (4) was converted to diselenide 5 by reaction with selenium powder followed by air oxidation. Treatment of $\mathbf{5}$ with two equivalents of nitrous acid gave dinitroso diselenide (6) as an orange solid. Attempts to condense $\mathbf{6}$ with $N$-ethyl-1-naphthylamine (7) in ethanol gave no reaction; increasingly better yields of EtNBSe (3) were realized in this solvent as progressively weaker acids, including $\mathrm{HCl}$, acetic acid, and acetic acid-sodium acetate, were used as the catalyst. This trend naturally led us to evaluate trifluoroethanol, which is mildly acidic and nonnucleophilic, as both solvent and benign catalyst, whereupon the desired dye was formed cleanly and in good yield. Treatment with an ion exchange procedure ensured that chloride was the counterion of $\mathbf{3}$; subsequent column chromatography afforded the chloride salt of EtNBSe (3) as a dark blue solid (55\% yield).

\section{Physical and Photophysical Properties}

An inspection of the molecular structures of the three chalcogen chloride dyes of the present study, presented in Figure 1, shows that they are relatively small, planar, and possess a delocalized positive charge that can be neutralized by the removal of the proton from the C-5 amino group; the distinguishing feature of each is the nature of the chalcogen atom residing at the 7-ring position. The generic molecular structure of the series is closely related to that of Nile Blue A, a commercial stain, differing by an additional ethyl moiety on the 5amino group; we discovered that the inclusion of this substituent greatly increased the rate of aqueous dissolubility while concomitantly increasing lipophilicity. We attribute this seeming paradox to added steric bulk that accompanies the incorporation of this additional alkyl group, which decreases the tendency of the dyes to form large, less water soluble aggregates. 
Physical and photophysical data pertinent to this work is presented in Table 1. All three dyes are efficient absorbers of red light with extinction coefficients greater than $50000 \mathrm{~L} / \mathrm{mol}$ $\mathrm{cm} ;{ }^{18}$ as expected, the wavelength of maximum absorption shifts to longer wavelengths as the chalcogen atom is varied from oxygen to sulfur and selenium. ${ }^{38}$ Photophysical data are presented for the dyes dissolved in ethanol rather than water because in aqueous media, all three chromophores form, in addition to monomeric species, H-dimers and possibly higher order aggregates, which we deduced from the appearance of an additional absorption band in the short red spectral region (data not shown); in ethanol, the dyes appear to be entirely monomeric as evidenced by their adherence to Beer's Law and the absence of the short red band. ${ }^{39}$ All three chalcogen chromophores fluoresce, with wavelengths of maximum emission shifted approximately $40 \mathrm{~nm}$ to the red of their respective absorbance maxima. This relationship is illustrated in Figure 2, which provides an example of the absorption and emission curves for selenium dye 3 ; the other two members of the series have similarly shaped spectral profiles.

The relative photoactivities of the three PSs studied herein were gauged by their abilities to generate singlet oxygen $\left({ }^{1} \mathrm{O}_{2}\right)$ when illuminated with a beam of red light with an adjusted intensity such that each dye absorbed the same number of photons per unit of time. As shown in Table 1, the quantum yields of ${ }^{1} \mathrm{O}_{2}$ formation, measured using the 1,3diphenylisobenzofuran method, rose dramatically as the atomic number of the chalcogen atom, and hence the spin-orbital coupling constants, increased from $\mathrm{O}$ to Se.

Finally, although previously presented elsewhere, ${ }^{18}$ because of the relevance of lipophilicity to the present studies, we list partition coefficients for the PS between 2-octanol and phosphate-buffered saline $(\mathrm{pH}=7.4)$.

\section{Antimicrobial Results}

\section{Photodynamic Inactivation of $E$. faecalis}

The Gram-positive bacterium E. faecalis was killed by all three PSs at a concentration of 2 $\mu \mathrm{M}$ followed by a wash and application of red light, but there were large and significant differences between the degrees of killing observed (Figure 3). Because preliminary experiments showed that the dyes had very different activities, we used more light than that used for EtNBSe (3, up to $\left.32 \mathrm{~J} / \mathrm{cm}^{2}\right)$ to kill cells incubated with EtNBS (2, up to $\left.64 \mathrm{~J} / \mathrm{cm}^{2}\right)$ and EtNBA (1, up to $\left.80 \mathrm{~J} / \mathrm{cm}^{2}\right)$. EtNBSe (3) was by far the most effective PS, leading to the killing of $>99.999 \%$ of cells $(5.5 \operatorname{logs}, P<0.001)$ after a delivery of only $8 \mathrm{~J} / \mathrm{cm}^{2}$ of $660-\mathrm{nm}$ light; EtNBS (2) was the next most efficient antimicrobial PS with a killing of $>99.9 \%$ (3 logs) after a delivery of $64 \mathrm{~J} / \mathrm{cm}^{2}$ of 652-nm light, whereas EtNBA (1) was the least effective PS, requiring the delivery of $80 \mathrm{~J} / \mathrm{cm}^{2}$ of $635-\mathrm{nm}$ light to kill $95 \%$ of the cells.

\section{Photodynamic Inactivation of $E$. coli}

We used a somewhat higher concentration $(5 \mu \mathrm{M})$ of PS to test the PDT killing of the Gramnegative $E$. coli after a wash. This was because Gram-negative species are generally found to be more resistant to PDT than Gram-positive species. ${ }^{20}$ Again EtNBSe (3) was by far the most effective PS with a delivery of $32 \mathrm{~J} / \mathrm{cm}^{2}$ giving almost total eradication of $>99.9999 \%$ (>6 logs, $P<0.001$ ) (Figure 4). EtNBS (2) was much less effective, leading to a killing of slightly more than $99 \%$ after $64 \mathrm{~J} / \mathrm{cm}^{2}$, whereas EtNBA (1) was again the least effective, only killing $90 \%$ of cells after $80 \mathrm{~J} / \mathrm{cm}^{2}$.

\section{Photodynamic Inactivation of $\boldsymbol{C}$. albicans}

Because Candida cells are 10 times larger than bacterial cells, ${ }^{22}$ we used an even higher PS concentration than that used for bacterial cells, that is, $20 \mu \mathrm{M}$ with a wash. Under these 
conditions, EtNBSe (3) was again the most effective PS by a large margin with only $4 \mathrm{~J} / \mathrm{cm}^{2}$ required to kill more than $99 \%$ of cells $(P<0.001)$ (Figure 5). EtNBS $(2)$ was less effective, needing a fluence of $64 \mathrm{~J} / \mathrm{cm}^{2}$ to give the same degree of cell killing (>98\%), whereas EtNBA (1) was the least effective requiring $80 \mathrm{~J} / \mathrm{cm}^{2}$ to kill $>97 \%$ of cells.

\section{Effect of Microorganism Wash Pre-PDT}

To test whether the PSs were more or less effective at killing microbial cells when present in solution during illumination, we compared three sets of experiments with and without a wash of the unbound PS from the cell suspension. The killing curves for E. faecalis incubated with $2 \mu \mathrm{M}$ EtNBSe (3), E. faecalis incubated with $2 \mu \mathrm{M}$ EtNBS (2), and E. coli incubated with $5 \mu \mathrm{M}$ of EtNBSe (3), all with and without a wash, are presented in the Supporting Information section. In all three cases, the two killing curves for wash and no wash are almost identical.

\section{Microbial Uptake}

We asked whether the observed large differences in effectiveness of antimicrobial PDT of the three dyes depended on photochemical efficiencies, the degree of cell-dye binding, or both. We therefore examined the uptake of dye in terms of molecules per cell by carrying out 10 min incubations with the same concentration of dyes that was used for photoinactivation followed by a wash to remove residual incubating solution. The resulting bacterial pellets were dissolved in 10\% SDS to give a homogeneous solution and comparison of the resulting fluorescence intensities with a calibration curve prepared with known concentrations of dye in the same solvent yielded the amount of dye in the pellet. Figure 6A shows that for E. faecalis the cell uptake was in the order EtNBSe (3) $>$ EtNBS (2) > EtNBA (1). The differences between EtNBA (1) and both EtNBS (2) and EtNBSe (3) were significant, but the difference between EtNBS (2) and EtNBSe (3) was not significant. A similar order of cell uptake (but higher values due to higher dye concentrations being used) was found for $E$. coli, with EtNBSe (3) $>$ EtNBS (2) > EtNBA (1); in this case, the differences between all three values were significant. For the fungus $C$. albicans, we used an even higher concentration of dye $(20 \mu \mathrm{M})$ because the eukaryotic cells are 10 times bigger than the prokaryotic bacterial cells. The uptake of EtNBA (1) was significantly lower than that found for EtNBSe (3).

By comparing the amount of dye that remained in solution with the dye that was in the bacterial pellet, it was possible to calculate the fraction of total dye added that was bound to the cells as shown in Figure 6B. The binding of EtNBSe (3) to C. albicans and E. coli and the binding of EtNBS (2) to C. albicans were essentially quantitative, whereas approximately $75 \%$ of the dye was cell bound in the case of EtNBA (1) to C. albicans, EtNBS (2) to E. coli, and EtNBSe (3) to E. faecalis. Thus, the affinity of dyes for cells increased in the order EtNBA (1) $<$ EtNBS $(\mathbf{2})<\operatorname{EtNBSe}(\mathbf{3})$, and the affinity of cells for dyes increased in the order E. faecalis $<E$. coli $<$ C. albicans.

\section{Discussion}

The evolution of disease causing bacteria into phenotypes that are becoming increasingly resistant to even the most powerful antibiotic drugs has provided the impetus for the discovery of new approaches for controlling pathogens. In this study, we show that a series of chalcogen-substituted phenoxazinium derivatives originally developed as anticancer PDT agents are also effective broad-spectrum antibacterial PSs. The physical and photophysical attributes that characterize the series and make these dyes well suited for this purpose are as follows. (1) They are strong absorbers of red light; (2) when illuminated with red photons, they become, or in reaction with molecular oxygen generate, a cytotoxin; (3) they are 
lipophilic and bear a delocalized positive charge; (4) they are relatively stable to photodegradation when illuminated in alcohol at moderate light fluences; and (5) they emit red or near-infrared fluorescence. Additionally, they are water soluble, which is beneficial for drug administration purposes, and two members of the series, $\mathbf{1}$ and $\mathbf{2}$, evaluated in prior extensive animal model studies, appear to have little or no toxicity in the dark.

The ability for an antimicrobial PS to absorb light in the $600-900 \mathrm{~nm}$ region of the spectrum is critically important for in vivo applications because these are the wavelengths that penetrate tissues most effectively. All three chalcogen analogues investigated herein meet this criterion with the oxygen-, sulfur-, and selenium-substituted PSs having absorption maxima at 634,653, and $661 \mathrm{~nm}$, respectively.

Photosensitizers usually inactivate biological targets via their excited triplet states primarily because the millisecond lifetimes of this species are long enough to participate in bimolecular chemistry, whereas the nanosecond lifetimes of the corresponding excited singlet state generally precludes this behavior. A triplet PS can inactivate biomolecules in one of two ways: (1) it can react via an electron transfer reaction directly with the target or with some other species to produce cytotoxic radicals, or (2) it can transfer its electronic energy to ground state oxygen to generate highly cytotoxic singlet oxygen, ${ }^{1} \mathrm{O}_{2}$, with regeneration of the ground-state dye. ${ }^{40}$ Regardless of which mechanism a PDT agent employs, an efficient PS must have a high triplet quantum yield. Because it is well established that in aerated alcoholic solvents the triplet states of phenothiazine dyes such as those studied herein generate singlet oxygen with $100 \%$ efficiencies $^{33}$ owing to their $\pi-\pi^{*}$ character, we chose to determine the triplet quantum yields of EtNBA (1), EtNBS (2), and EtNBSe (3) indirectly by measuring their respective singlet oxygen quantum yields in ethanol. EtNBA has an extremely low triplet yield (0.003), which is not surprising because this dye lacks any of the structural features that promote intersystem crossing. Because it is well-known that the incorporation of a heavy atom into a molecule with a low intrinsic intersystem crossing rate constant will increase the probability of such transitions roughly in proportion to the square of the spin-orbital coupling constant $(\zeta)$ of the atom where the transition occurs, ${ }^{41}$ ignoring the small contributions from $\mathrm{C}$ and $\mathrm{N}\left(\zeta=13,76 \mathrm{~cm}^{-1}\right.$, respectively), we found that replacing the central $\mathrm{O}$ atom $\left(\zeta=-79.6 \mathrm{~cm}^{-1}\right)$ with a somewhat heavier $\mathrm{S}$ atom $\left(\zeta=-184 \mathrm{~cm}^{-1}\right)$ resulted in a small but significant, increase in the triplet yield $(0.03)$ and that as expected the replacement by a much heavier Se atom $(\zeta=-825$ $\left.\mathrm{cm}^{-1}\right)^{42}$ resulted in a dramatic improvement in the triplet yield (0.78); these values are similar to results previously obtained in methanol. ${ }^{18}$

On the basis of an extensive study of Gram-positive and Gram-negative antibacterial agents, Hansch et. al. concluded that the most important factor in determining activity was lipophilic character as expressed by their oil-water partition coefficients. ${ }^{43}$ With this in mind, in Table 1 , we list partition coefficient data showing that all three chalcogen dyes are lipophilic, with values greater than $\log 2$. In comparison, under identical conditions, the PDT antibacterial drug Methylene Blue has a $\log P=0 ;{ }^{44}$ the difference in lipophilicity between the dyes of this study and Methylene Blue might explain the superior microbial affinities of the former materials as reflected in chromophore washing studies (vide infra).

Because most PDT agents emit a fluorescent signal upon excitation with visible light, they offer the valuable benefit of doubling as real-time in situ luminescent reporters of the location and concentration of the drug. Such is the case with the PSs of the present investigation; $\mathbf{1}$ and $\mathbf{2}$ are strong emitters that can easily be detected with a fluorescence microscope, whereas $\mathbf{3}$, because of its relatively low fluorescence quantum yield, is more challenging to detect visually. Nevertheless, even selenium analogue $\mathbf{3}$ emits with an 
intensity that can easily be detected by modern electronic imaging instrumentation of the type used in our quantitative studies.

The microorganism species chosen to evaluate the photoactivities of the dyes in the present study are important pathogens in the type of infections, such as wounds and burns, likely to be amenable to PDT. E. faecalis is typical of Enterococci that frequently develop vancomycin resistance and are important nocosomial pathogens in postsurgical wounds and burns. ${ }^{45}$ Although E. coli is generally thought of as an intestinal or urinary tract pathogen, there is increasing concern about its presence in wound infections, and its ability to develop extended spectrum beta-lactamase resistance is causing increased international concern. 46,47 E. coli is generally considered to be a good model Gram-negative organism to test PDT because it is midway in susceptibility between the easy to kill Hemophilus and the difficult to kill Pseudomonas. C. albicans is increasingly found in nocosomial infections in burns ${ }^{48}$ and to a lesser extent in surgical wounds. ${ }^{49}$

When evaluated against these prototypical cell types, EtNBSe (3) was found to be a highly effective, broad-spectrum antimicrobial PDT drug. It was active against Gram-positive and Gram-negative bacterial and fungal cells and was equally effective whether or not the cells were washed of free dye. A possible reason for this latter observation is the almost quantitative binding of the dye to the cells after a 10 min incubation (75-98\%). Cell-dye binding was also found to be stronger for EtNBS (2) than for EtNBA (1). As note above, phenothiazinium dyes such as Toluidine Blue $\mathrm{O}$ and Methylene Blue have previously been used as antimicrobial PSs. However, much higher concentrations of these dyes must be used to achieve the degree of microorganism inactivation found using selenium analogue 3 . The reason this higher concentration of dye is needed for phenothiazinium salts is probably the fact that the binding between the dye and the cell is weaker and that the cell uptake is correspondingly lower. In addition, it has been found that PDT using Methylene Blue and Toluidine Blue to kill Gram-positive and Gram-negative bacteria and fungi is much more effective when the free dye of the incubating solution is left in the cell suspension than when it is washed out. This observation may be explained by the fact that the dye that remains in solution can generate reactive oxygen species in the vicinity of the microbial cells that can damage the outer structures of the cell, directly causing cell death, or by allowing more dye to bind or penetrate the organism, thus accelerating the PDT effect.

The high efficiency of EtNBSe (3) compared to that found for EtNBS (2) and EtNBA (1) is presumably due to the much-increased yields of an excited triplet state species, which can be cytotoxic in its own right or can react with ubiquitous molecular oxygen generating singlet oxygen or superoxide, depending on whether the reaction occurs via energy or electron transfer, respectively. This conclusion is supported by the observation that although uptake by the cells was slightly higher for EtNBSe (3) compared to that for EtNBS (2), there is a very large difference in killing efficacy between the two (2-5 logs depending on cell type). Significantly, EtNBSe (3) killed 5 logs more of the Gram-negative E. coli than EtNBS (32 J/ $\mathrm{cm}^{2}$ ), and it is well-known that Gram-negative bacteria are, in general, most resistant to antimicrobial PDT. ${ }^{19-21}$

\section{Conclusion}

A benzo[ $a]$ phenoselenazinium dye (3) has been found to be an effective broad-spectrum antimicrobial PDT agent in vitro. The dye has many of the characteristics that constitute the hallmarks of an effective antibacterial PS: it absorbs red light efficiently, it binds strongly to both Gram-positive and Gram-negative bacteria as well as Candida fungi, it is highly phototoxic to these pathogens, and retains this level of activity even under washing conditions. The high photodynamic activity and the broad-spectrum of targets inactivated by 
EtNBSe (3) supports further testing of this novel antimicrobial PS in animal models of localized infections.

\section{Acknowledgments}

J.W.F. and X.S. were supported by the Rowland Institute at Harvard University. F.J. and M.R.H. were funded by the National Institute of Allergy and Infectious Disease (grant number R01AI050875 to M.R.H.). T.N.D. was supported by a Wellman Center of Photomedicine graduate student fellowship.

\section{References}

(1). Dougherty TJ. Photosensitizers: Therapy and Detection of Malignant-Tumors. Photochem. Photobiol. 1987; 45:879-889. [PubMed: 2957705]

(2). Castano AP, Demidova TN, Hamblin MR. Mechanisms in photodynamic therapy: part onephotosensitizers, photochemistry and cellular localization. Photodiagnosis Photodyn. Ther. 2004; 1:279-293.

(3). Castano AP, Demidova TN, Hamblin MR. Mechanisms in photodynamic therapy: part twocellular signalling, cell metabolism and modes of cell death. Photodiagnosis Photodyn. Ther. 2005; $2: 1-23$.

(4). Castano AP, Demidova TN, Hamblin MR. Mechanisms in photodynamic therapy: part three:photosensitizer pharm-pocokinetics, biodistribution, tumor localization and modes of tumor destruction. Photodiagnosis Photodyn. Ther. 2005; 2:91-106.

(5). Detty MR, Gibson SL, Wagner SJ. Current clinical and preclinical photosensitizers for use in photodynamic therapy. J. Med. Chem. 2004; 47:3897-3915. [PubMed: 15267226]

(6). Michl, J.; Bonacic-Koutecky, V. Electronic Aspects of Organic Photochemistry. 1st ed.. John Wiley \& Sons; New York: 1990.

(7). Moan J, Peng Q. An outline of the hundred-year history of PDT. Anticancer Res. 2003; 23:35913600. [PubMed: 14666654]

(8). Dolmans D, Fukumura D, Jain RK. Photodynamic therapy for cancer. Nat. Rev. Cancer. 2003; 3:380-387. [PubMed: 12724736]

(9). Lin C-W. Photodynamic therapy of malignant tumors: recent developments. Cancer Cells (Cold Spring Harbor). 1992; 3:437-444.

(10). Henderson BW, Dougherty TJ. How does photodynamic therapy work? Photochem. Photobiol. 1992; 55:145-157. [PubMed: 1603846]

(11). Lin CW, Shulok JR, Wong YK, Schanbacher CF, Cincotta L, Foley JW. Photosensitization, uptake, and retention of phenoxazine nile blue derivatives in human bladder-carcinoma cells. Cancer Res. 1991; 51:1109-1116. [PubMed: 1847656]

(12). Cincotta L, Foley JW, Cincotta AH. Novel red absorbing benzo[a]phenoxazinium and benzo[ $a]$ phenothiazinium photosensitizers: In vitro evaluation. Photochem. Photobiol. 1987; 46:751-758. [PubMed: 3441499]

(13). Lewis MR, Sloviter HA, Goland PP. In vivo staining and retardation of growth of sarcomata in mice. Anat. Rec. 1946; 95:89-96. [PubMed: 20990816]

(14). Lewis MR, Goland PP, Sloviter HA. The Action of Oxazine Dyes on Tumors in Mice. Cancer Res. 1949; 9:736-740. [PubMed: 15407653]

(15). Cincotta AH, Cincotta L, Foley JW. Novel benzophenothiazinium photosensitizers: preliminay in vivo results. SPIE. 1990; 1203:202-210.

(16). Cincotta L, Foley JW, Maceachern T, Lampros E, Cincotta AH. Novel photodynamic effects of a benzophenothiazine on 2 different murine sarcomas. Cancer Res. 1994; 54:1249-1258. [PubMed: 8118813]

(17). Frimberger AE, Moore AS, Cincotta L, Cotter SM, Foley JW. Photodynamic therapy of naturally occurring tumors in animals using a novel benzophenothiazine photosensitizer. Clin. Cancer Res. 1998; 4:2207-2218. [PubMed: 9748141] 
(18). Cincotta L, Foley JW, Cincotta AH. Phototoxicity, redox behavior, and pharmacokinetics of benzophenoxazine analogs in Emt-6 murine sarcoma-cells. Cancer Res. 1993; 53:2571-2580. [PubMed: 8495421]

(19). Hamblin MR, Hasan T. Photodynamic therapy: A new antimicrobial approach to infectious disease? Photochem. Photobiol. Sci. 2004; 3:436-450. [PubMed: 15122361]

(20). Malik Z, Ladan H, Nitzan Y. Photodynamic inactivation of Gram-negative bacteria: Problems and possible solutions. J. Photochem. Photobiol., B. 1992; 14:262-266. [PubMed: 1432395]

(21). Nitzan Y, Gutterman M, Malik Z, Ehrenberg B. Inactivation of Gram-negative bacteria by photosensitized porphyrins. Photochem. Photobiol. 1992; 55:89-96. [PubMed: 1534909]

(22). Demidova TN, Hamblin MR. Effect of cell-photo sensitizer binding and cell density on microbial photoinactivation. Antimicrob. Agents Chemother. 2005; 49:2329-2335. [PubMed: 15917529]

(23). Merchat M, Bertolini G, Giacomini P, Villanueva A, Jori G. Meso-substituted cationic porphyrins as efficient photosensitizers of gram-positive and gram-negative bacteria. J. Photochem. Photobiol., B. 1996; 32:153-157. [PubMed: 8622178]

(24). Voet, D.; Voet, JG. Biochemistry. 1st ed.. John Wiley \& Sons; New York: 1990.

(25). Hamouda T, Shih AY, Baker JR. A rapid staining technique for the detection of the initiation of germination of bactyerial spores. Lett. Appl. Microbiol. 2002; 34:86-90. [PubMed: 11849500]

(26). Phoenix DA, Harris F. Phenothiazinium-based photosensitizers: antibacterials of the future? Trends Mol. Med. 2003; 9:283-285. [PubMed: 12900214]

(27). 2005. http://www.ondinebiopharma.com/periodontal_disease_therapy.php

(28). 2005. http://www.denfotex.com/

(29). Foley, JW.; Cincotta, L. Photo-inactivation of Cancer Cells. U.S. Patent 4,962,197. 1990.

(30). Nikas DC, Foley JW, Black PM. Fluorescent imaging in a glioma model in vivo. Lasers Surg. Med. 2001; 29:11-17. [PubMed: 11500856]

(31). Clapp RC, English JP, Fellows CE, Forsythe J, Grotz RE, Shepherd RG. Chemotherapeutic dyes. 5. Benzo $[a]$ phenothiazines and benzo[a]phenazines. J. Am. Chem. Soc. 1952; 74:1994-1996.

(32). Magde D, Brannon JH, Cremers TL, Olmsted J. Absolute luminescence yield of cresyl violet: Standard for the red. J. Phys. Chem. 1979; 83:696-699.

(33). Tanielian C, Wolff C, Esch M. Singlet oxygen production in water: Aggregation and chargetransfer effects. J. Phys. Chem. 1996; 100:6555-6560.

(34). Gregory P, Thorp D. Electronic absorption-spectra of 2-substituted-4-Nn-diethylamino-4'nitroazobenzene dyes and their monoacid cations: Applicability of Dewar rules to these and related dyestuffs. J. Chem. Soc., Perkin Trans. 1. 1979; 8:1990-2000.

(35). Griffiths, J. Colour and Constitution of Organic Molecules. 1st ed.. Acedemic Press; New York: 1976. p. 264

(36). Jett BD, Hatter KL, Huycke MM, Gilmore MS. Simplified agar plate method for quantifying viable bacteria. Biotechniques. 1997; 23:648-650. [PubMed: 9343684]

(37). Groves JT, Lindenau SM, Haywood BJ, Knol JA, Schultz JS. Synthesis of seleno-toluidine blue. J. Med. Chem. 1974; 17:902-904. [PubMed: 4845385]

(38). Detty MR, Merkel PB. Chalcogenapyrylium dyes as potential photochemotherapeutic agents: Solution studies of heavy-atom effects on triplet yields, quantum efficiencies of singlet oxygen generation, rates of reaction with singlet oxygen, and emission quantum yields. J. Am. Chem. Soc. 1990; 112:3845-3855.

(39). Bergmann K, O'Konski CT. A spectroscopic study of methylene blue monomer, dimer, and complexes with monillonite. J. Phys. Chem. 1963; 67:2169-2175.

(40). Henderson BW, Miller AC. Effects of scavengers of reactive oxygen and radical species on cellsurvival following photodynamic treatment in vitro: Comparison to ionizing-radiation. Radiat. Res. 1986; 108:196-205. [PubMed: 3097749]

(41). Hamanoue K, Nakayama T, Tsujimoto I, Miki S, Ushida K. Further studies on the nature of the lowest excited triplet states of haloanthraquinones: triplet-triplet absorption and phosphorescence spectra of the $\alpha$-halo(1-bromo,1-bromo-5-chloro,1,5-dibromo,1-bromo-8-chloro, 1,8-dibromo) and $\beta$-halo(2-bromo) compounds. J. Phys. Chem. 1995; 99:5802-5808. 
(42). Atkins, PW. Quanta, A Handbook of Concepts. 2nd ed.. Oxford University Press; Oxford, England: 1991. p. 339

(43). Lien EJ, Hansch C, Anderson SM. Structure-activity correlations for antibacterial agents on gram-positive and gram-negative cells. J. Med. Chem. 1968; 11:430-441. [PubMed: 5656475]

(44). Cincotta L, Foley JW, Cincotta AH. Novel phenothiazinuium photosensitizers for photodynamic therapy. SPIE. 1988; 997:145-153.

(45). Broder KW, Moise PA, Schultz RO, Forrest A, Schentag JJ. Clinical experience with linezolid in conjunction with wound coverage techniques for skin and soft-tissue infections and postoperative osteomyelitis. Ann. Plastic Surgery. 2004; 52:385-390.

(46). Russo TA, Johnson JR. Medical and economic impact of extraintestinal infections due to Escherichia coli: Focus on an increasingly important endemic problem. Microbes Infect. 2003; 5:449-456. [PubMed: 12738001]

(47). Shinagawa N, Hirata K, Katsuramaki T, Hata F, Ushijima Y, Ushida T, Tanimura H, Taniguchi K, Aikawa N, Yo K, Yamaue H, Ohnishi H, Takayama T, Sato T, Kato K, Tanaka N, Iwagaki H, Yura J, Fuchimoto S, Manabe T, Takeyama H, Kimura H, Hasegawa M, Takeuchi H, Yasui Y, Taniguchi M, Sueda T, Takesue Y, Mashita K, Hiyama E, Ishikawa S, Yokoyama T, Mizuno A, Ikeda S, Yasunami Y, Kubo S, Takada N, Suzuki Y, Fujimoto M. Bacteria isolated from surgical infections and its susceptibilities to antimicrobial agents-special references to bacteria isolated between April 2003 and March 2004. Jpn. J. Antibiot. 2005; 58:123-158. [PubMed: 15997655]

(48). Still JM, Law EJ, Belcher KE, Spencer SA. A Comparison of susceptibility to 5 antifungal agents of yeast cultures from burn patients. Burns. 1995; 21:167-170. [PubMed: 7794495]

(49). Chen YC, Chang SC, Sun CC, Yang LS, Hsieh WC, Luh KT. Secular trends in the epidemiology of nosocomial fungal infections at a teaching hospital in Taiwan, 1981 to 1993. Infection Control and Hospital Epidemiology. 1997; 18:369-375. [PubMed: 9154483] 


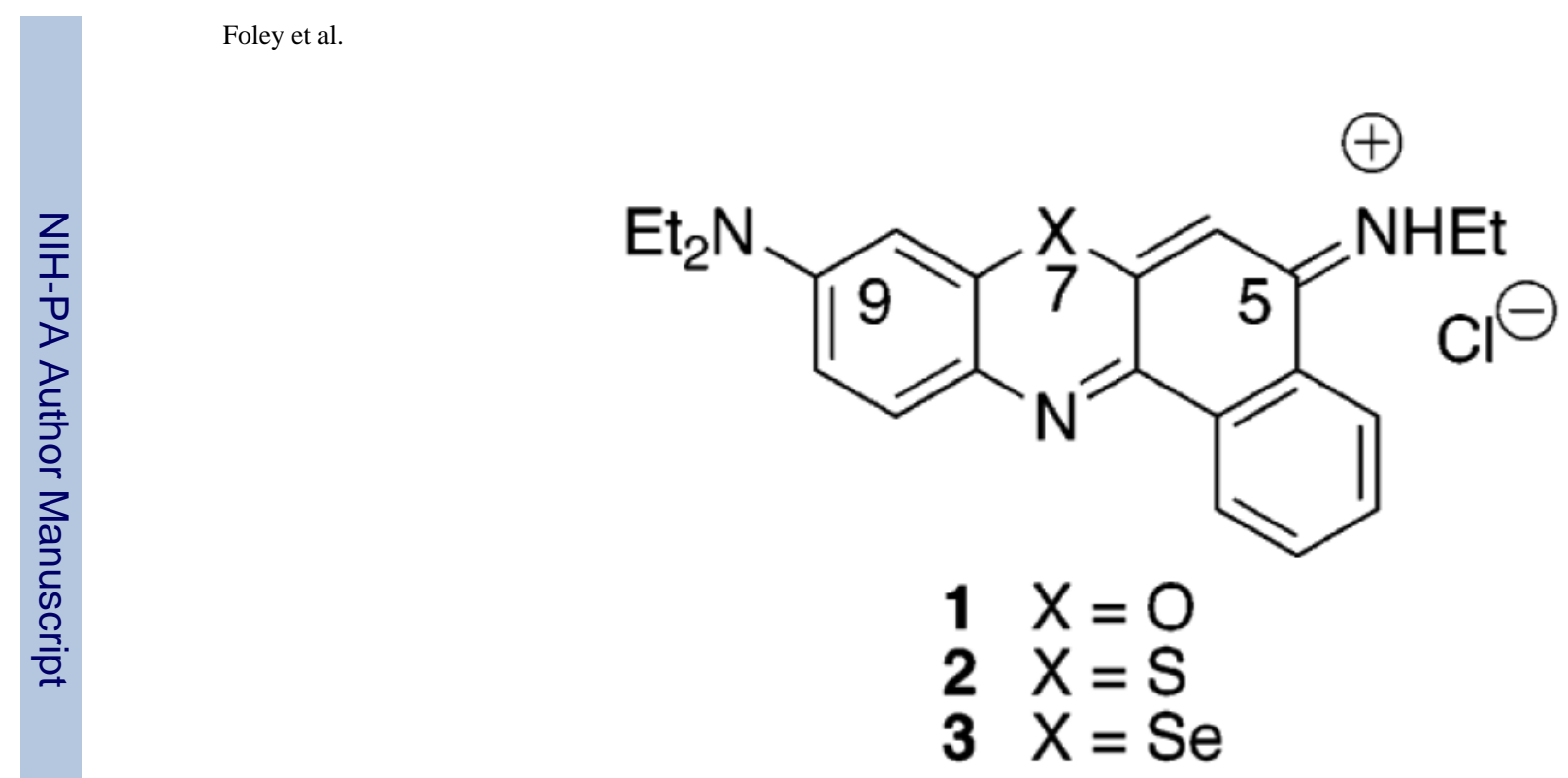

Figure 1.

Molecular structures of chalcogen-substituted photosensitizers. 


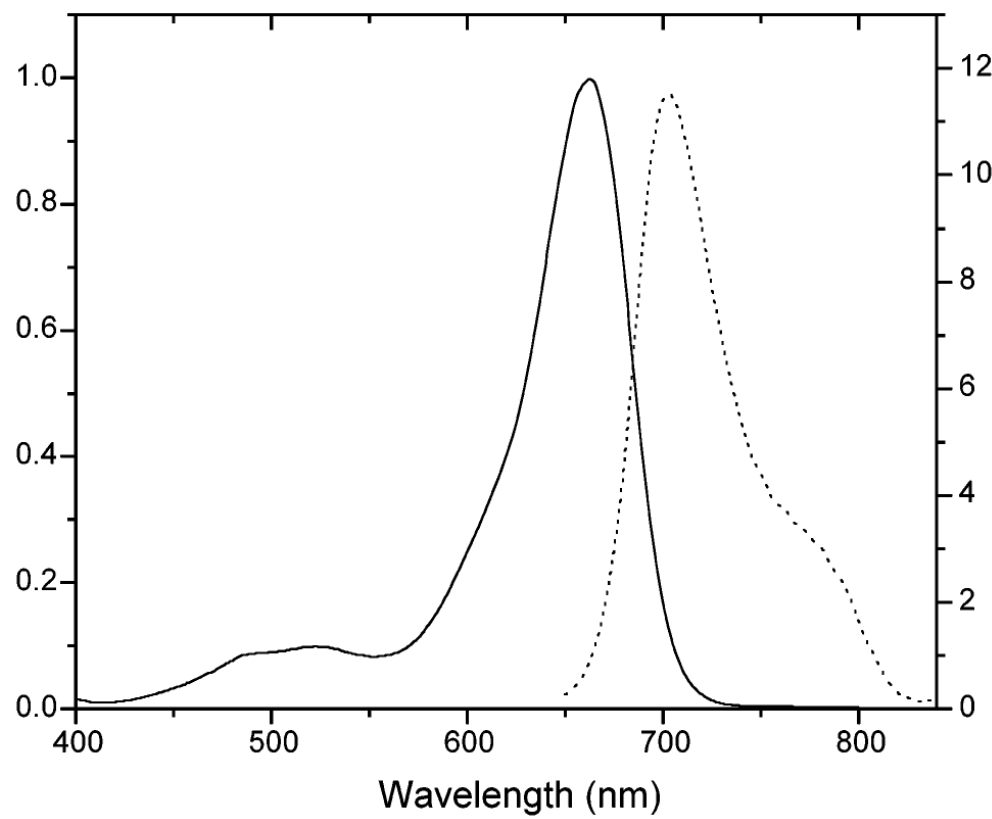

Figure 2.

Absorption and fluorescence emission spectra of selenium analogue $\mathbf{3}$ in ethanol. 


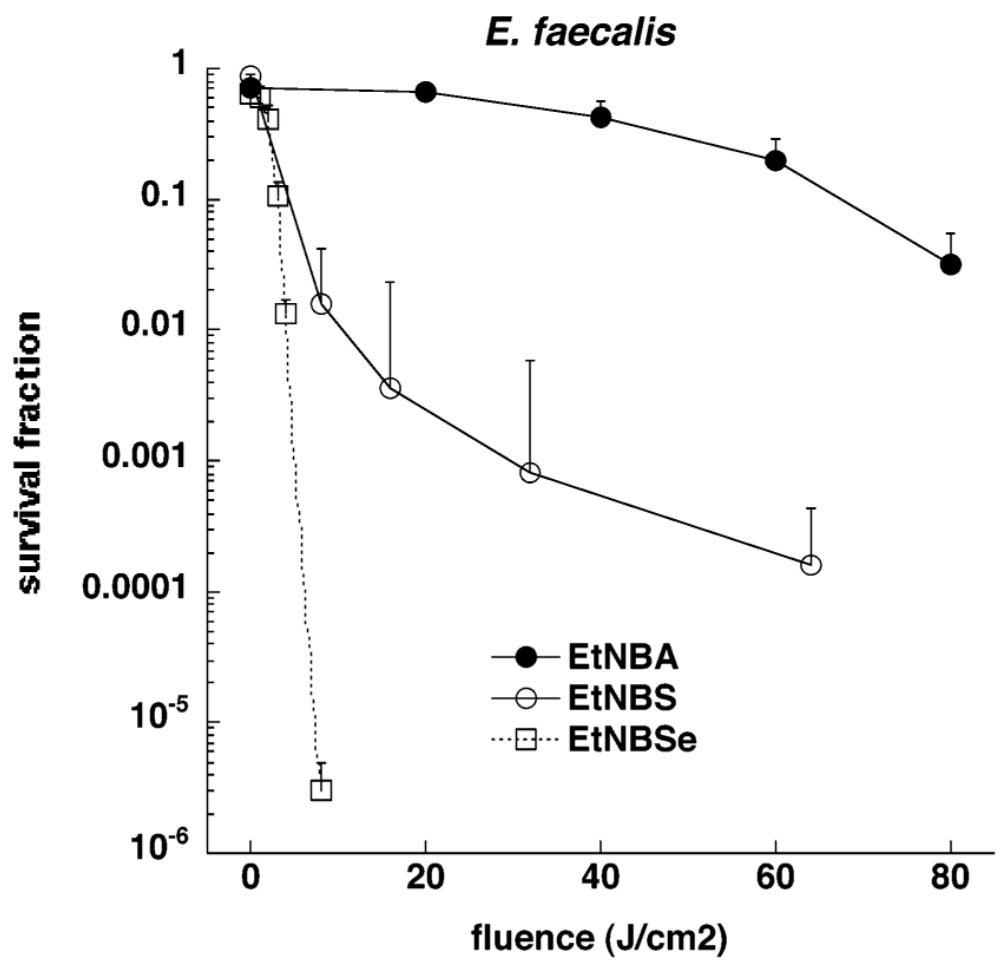

Figure 3.

Photodynamic inactivation of the Gram-positive E. faecalis (cell density $10^{8} / \mathrm{mL}$ ). Bacteria were incubated for $10 \mathrm{~min}$ with $2 \mu \mathrm{M}$ dyes, followed by a wash and illumination with the appropriate wavelength of red light. The values are means of three independent experiments, and the bars are SD. 


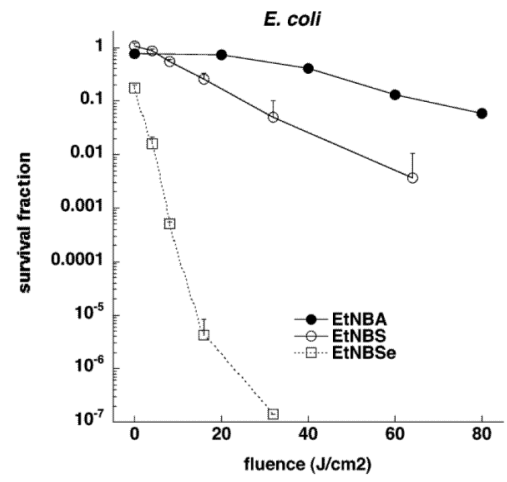

Figure 4.

Photodynamic inactivation of Gram-negative E. coli (cell density $10^{8} / \mathrm{mL}$ ). Bacteria were incubated for $10 \mathrm{~min}$ with $5 \mu \mathrm{M}$ dyes, followed by a wash and illumination with red light. The values are means of three independent experiments, and the bars are SD. 


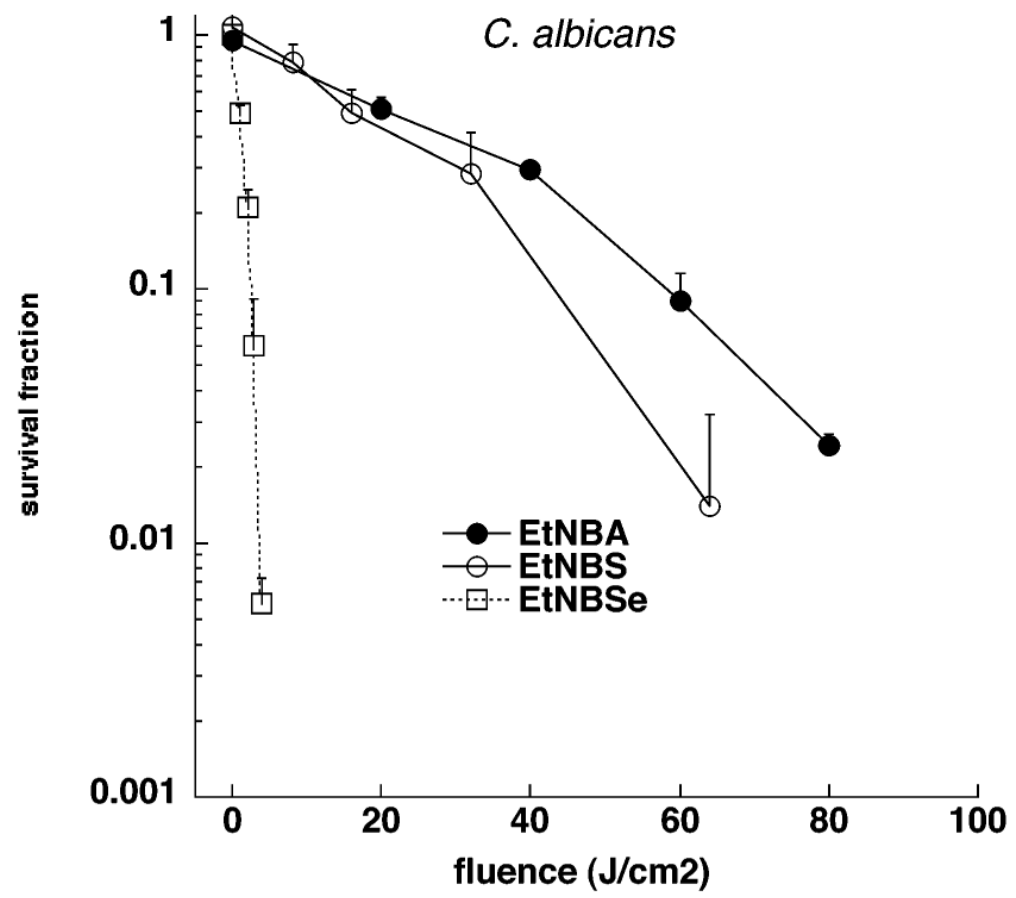

Figure 5.

Photodynamic inactivation of the pathogenic yeast C. albicans (cell density $10^{8} / \mathrm{mL}$ ) incubated for 10 min with $20 \mu \mathrm{M}$ dyes, followed by a wash and illumination with red light. The values are means of three independent experiments, and the bars are SD. 

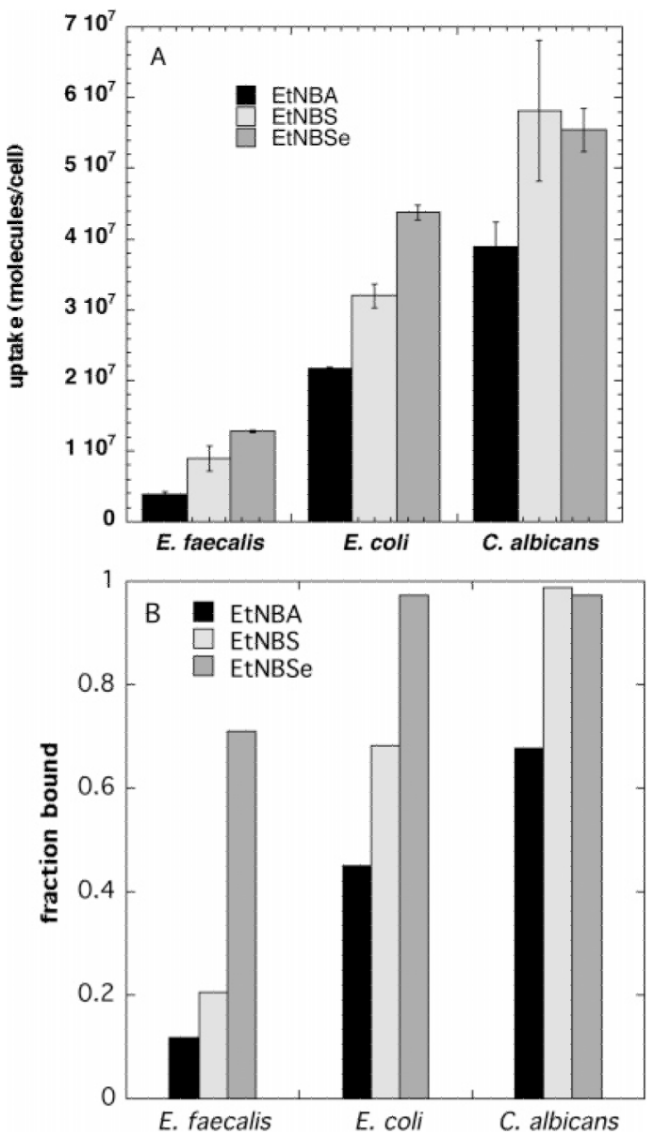

Figure 6.

(A) Uptake of the three PSs by the three microbial species. The concentration was $2 \mu \mathrm{M}$ for $E$. faecalis, $5 \mu \mathrm{M}$ for $E$. coli, and $20 \mu \mathrm{M}$ for C. albicans. Cells were incubated for $10 \mathrm{~min}$ followed by a wash and dissolving the pellet in $10 \%$ SDS and fluorescence quantification. The values are means of three independent experiments, and the bars are SD. ${ }^{*}, * *, * * *, P$ $<0.05,0.01,0.001$, respectively vs EtNBA (1); \#\# $P,<0.01$ vs 1. (B) Fraction of added PS that is bound to cells. Conditions as in Figure 6A. Fluorescence was determined in the dissolved bacterial pellet and in the remaining in supernatant. 

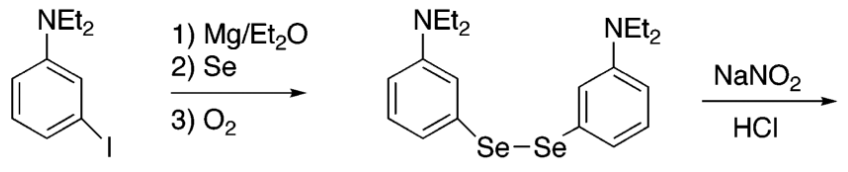<smiles>CC=Nc1ccc([N+](=O)[O-])c([Se][Se][N+](=O)c2ccc(NCC)cc2CC)c1</smiles>

6

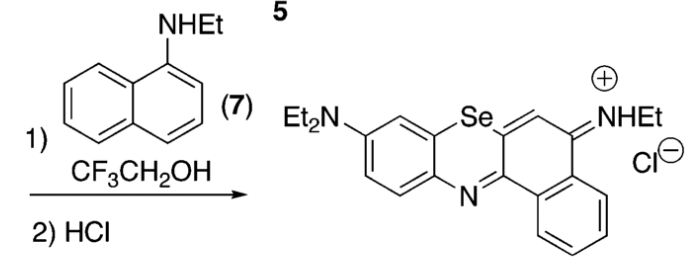

3

Scheme 1. 
Table 1

Physical and Photophysical Properties of Pertinent Dyes

\begin{tabular}{cccccc}
\hline dye & $\begin{array}{c}\lambda_{\mathrm{abs}} \\
(\mathbf{n m})^{\boldsymbol{a}}\end{array}$ & $\begin{array}{c}\lambda_{\mathrm{fl}} \\
(\mathbf{n m})^{b}\end{array}$ & $\boldsymbol{\Phi}_{\mathrm{fl}} \boldsymbol{c}$ & $\mathbf{1}_{\mathbf{O}_{2}} \boldsymbol{d}$ & $\log \boldsymbol{P}^{\boldsymbol{e}}$ \\
\hline 1 & 632 & 660 & 0.27 & 0.003 & 2.69 \\
2 & 654 & 693 & 0.21 & 0.03 & 2.76 \\
3 & 661 & 703 & 0.03 & 0.78 & 2.08 \\
\hline
\end{tabular}

${ }^{a}$ Absorption maximum in ethanol containing $0.1 \%$ acetic acid.

${ }^{b}$ Fluorescence maximum in ethanol.

${ }^{c}$ Absolute fluorescence quantum yield.

$d_{\text {Absolute quantum yield for singlet oxygen formation. }}$

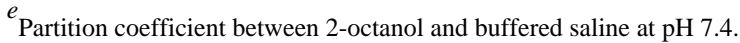

